\title{
Gesundheitsökonomie und ADHS I: Versorgung und Krankheitskosten
}

\begin{abstract}
Experten aus Deutschland widmeten sich in einer zweiteiligen Veröffentlichung der gesundheitsökonomischen Bewertung der Aufmerksamkeitsdefizit-/Hyperaktivitätsstörung (ADHS). Im ersten Teil ihrer Studie ging es um die Versorgungsepidemiologie sowie die Krankheitskosten.
\end{abstract}

\begin{abstract}
ie Autoren stellen im ersten Teil ihrer Untersuchung die Versorgungssituation in Deutschland vor: Administrativen Daten aus Nordbaden zufolge wird die Diagnose ADHS bei $5 \%$ aller 7-12-jährigen Kinder und bei 1,3\% aller 13-19-jährigen Jugendlichen gestellt. Wie auch aus internationalen epidemiologischen Studien bekannt, ist ein niedriger sozioökonomischer Status mit einer relativ höheren Häufigkeit an ADHS assoziiert. Die Autoren konnten außerdem belegen, dass nur ein Drittel der Diagnosen von auf Erkrankungen des zentralen Nervensystems spezialisierten Ärzten erhoben wird, was die bedeutende Rolle des Kinder- und Jugendarztes in der Diagnostik und Therapie der ADHS unterstreicht.
\end{abstract}

Die direkten Kosten für die Betreuung eines Kindes mit ADHS liegen um das 2,5-Fache höher als die Kosten für ein Kind ohne ADHS. 2003 dürften damit für die Krankenkassen zusätzliche Ausgaben von 260 Mio. Euro entstanden sein. Darüber hinaus ist die ADHS mit erheblichen indirekten Kosten durch Unfälle, Abhängigkeitserkrankungen, Strafvollzug, sekundären psychische Störungen, Arbeitslosigkeit etc. assoziiert. Darüber existieren in Deutschland keine Daten. Legt man jedoch amerikanische Berechnungen zugrunde, dürften sich die Zusatzkosten im Milliardenbereich bewegen.

Kommentar: Die erhebliche ökonomische Belastung sollte - abgesehen von der deutlich herabgesetzten Lebensqua- lität der Kinder und ihrer Familien - dazu motivieren, Kinder mit ADHS mit präventiven und frühzeitig beginnenden therapeutischen Maßnahmen zu begleiten. Dazu stellt der KBV-Vertrag ADHS, der im Mai 2008 von der Vertragswerkstatt der Kassenärztlichen Bundesvereinigung vorgestellt wurde, einen ersten wichtigen Schritt dar. Ermöglicht er doch erstmals, dass die „Hauptversorger“, die Kinder- und Jugendärzte, adäquat vergütet werden, wenn sie die Qualitätskriterien für Diagnostik und Therapie erfüllen. Leider wird der Vertrag bisher nur in Baden-Württemberg in Kooperation mit einer BKK umgesetzt. Wenn keine weiteren Abschlüsse folgen, wären die Folge für die Versorgung der Kinder mit ADHS wäre katastrophal: Zunehmend verlieren die Kinder- und Jugendärzte die Motivation, die zeitaufwendige Diagnostik und Therapie der ADHS als „Hobby“ zu betreiben.

Dr. Kirsten Stoffhoff

Schlander $M$ et al. Gesundheitsökonomie der ADHS in Deutschland: Teil 1: Versorgungsepidemiologie und Krankheitskosten Versorgungsepidemiologie. Nervenarzt 2010; 81: 289-300

\section{Gesundheitsökonomie und ADHS II: Kosteneffektivität der Therapie}

\section{Im zweiten Teil ihrer Publikation beschäftigen sich die Autoren mit den verschiedenen Therapieoptionen, die bei ADHS zur Verfügung stehen, und ihrer Kosteneffektivität.}

$\mathrm{D}$ ie Autoren ermitteln auf der Basis der Ergebnisse der MTA-Studie die Kosten der ADHS-Behandlung in Relation zu ihrer Effektivität. Sie kommen zu dem Schluss, dass eine medikamentöse Therapie unter intensiver Betreuung durch spezialisierte Ärzte nach weithin akzeptierten gesundheitsökonomischen Maßstäben kosteneffektiv ist und damit das Kriterium für Wirtschaftlichkeit erfüllt.

Auch unter Einschluss der neueren lang wirksamen Stimulanzienpräparate wird Kosteneffektivität erreicht. Die gesundheitsökonomische Bewertung von Atomoxetin fällt demgegenüber weniger überzeugend aus. Bei höheren Kosten und geringerer Effektstärke gegenüber lang wirksamem Methylphenidat muss für Atomoxetin von einer ökonomischen Inferiorität ausgegangen werden. Die derzeitige Datenlage lässt außerdem darauf schließen, dass eine allein psychotherapeutisch ausgerichtete Strategie unter dem Gesichtspunkt der Kosteneffektivität einer intensiven medikamentösen Therapie unterlegen sein dürfte.

Kommentar: Die medikamentöse Therapie, ärztlich gut betreut, ist nicht nur wirksamer, sie ist auch noch kostengünstiger als alle anderen Therapiemaßnahmen. Vor allem ist sie aber auch deshalb so kostengünstig, weil die medizinische Betreuung durch den Kinder- und Jugendarzt in Deutschland fast kostenfrei stattfindet - die Frage ist, wie lange noch.

Die Berechnungen der Autoren ziehen leider nicht die Kosten der jahrelang durchgeführten Ergotherapien mit ein. Es ist bekannt, dass hier Milliardenbeträge umgesetzt werden. Es bleibt nur zu spekulieren, wie hoch die Wirksamkeit einer Ergotherapie sein kann, wenn schon eine fachlich kompetent durchgeführte hochintensive Verhaltenstherapie - wie sie in der MTA-Studie stattfand - eine signifikant geringere Wirksamkeit aufwies als eine fachlich kompetent geleitete medikamentöse Therapie. Für den niedergelassen Arzt, der Kinder mit ADHS medikamentös betreut, sind die Berechnungen der Autoren sicher hilfreich, wenn er wieder einmal in eine Wirtschaftlichkeitsprüfung kommt.

Dr. Kirsten Stollhoff

Schlander M et al. Gesundheitsökonomie der ADHS in Deutschland: Teil 2: Therapeutische Optionen und ihre Kosteneffektivität. Nervenarzt 2010; 81: 301-14 